\title{
Controlling of Rotor Flux for Doubly Fed Induction Machine- Based Wind Turbines under Voltages Dips and Without Crowbar Protection
}

\author{
T. Muni Prakash ${ }^{1}$, S. K. Shakeer Ahammad ${ }^{2}$, V. Sekhar ${ }^{3}$ \\ ${ }^{I} P$ G SCHOLAR@KEC Department of EEE, KUPPAM \\ ${ }^{2}$ Asst.Professor@MeRITS Department of EEE, UDYAGIRI \\ ${ }^{3}$ Asst.Professor@KEC Department of EEE, KUPPAM
}

\begin{abstract}
This paper proposes a rotor flux amplitude reference generation strategy for doubly fed induction machine based wind turbines. It is designed to address perturbations, such as voltage dips, keeping controlled the torque of the wind turbine, and considerably reducing the stator and rotor over currents during faults. In addition, a direct torque control strategy that provides fast dynamic response accompanies the overall control of the wind turbine. Despite the fact that the proposed control does not totally eliminate the necessity of the typical crowbar protection for this kind of turbines, it eliminates the activation of this protection during low voltage dips.
\end{abstract}

Index Terms: Under voltage dips, Crowbar protection, direct torque control, doubly fed induction machine, reference flux generation strategy.

\section{Introduction}

This paper focuses the analysis on the control of doubly fed induction machine (DFIM) based high power wind turbines when they operate under presence of voltage dips. Most of the wind turbine manufacturers build this kind of wind turbines with back to back converter sized to approximately $30 \%$ of the nominal power [1]. This reduced converter design provokes that when the machine is affected by voltages dips, it needs a special crowbar protection [2] in order to avoid damages in the wind turbine and meet the grid code requirements.

The main objective of the control strategy proposed in this letter is to eliminate the necessity of the crowbar protection when a low-depth voltage dip occurs. Hence, by using direct torque control (DTC), with a proper rotor flux generation strategy, during the fault it will be possible to maintain the machine connected to the grid, generating power from the wind, reducing over currents, and eliminating the torque oscillations that normally produce such voltage dips. In Fig.1 the wind turbine generation system together with the proposed control block diagram is illustrated. The DFIM is supplied by a back-to-back converter through the rotor, while the stator is directly connected to the grid.

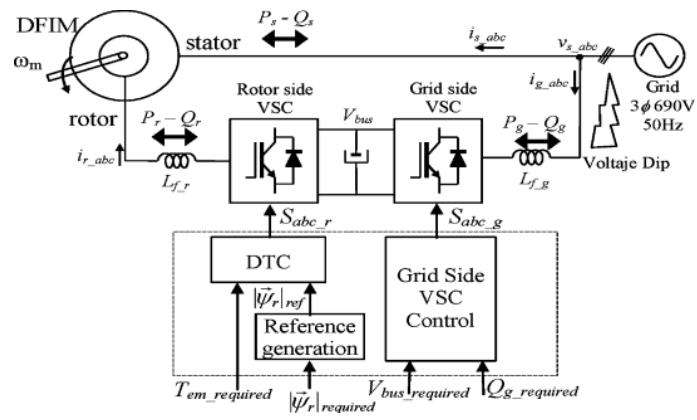

Fig.1.Wind energy generation system based on the DFIM

This paper only considers the control strategy corresponding to the rotor side converter. The grid side converter is in charge to keep controlled the dc bus voltage of the back-to back converter and reactive power is exchanged through the grid by this. As can be noticed from Fig.1 the DFIM control is divided into two different control blocks. A DTC that controls the machine's torque $\left(\mathrm{T}_{\mathrm{em}}\right)$ and the rotor flux amplitude $\left(\left|\overrightarrow{\Psi_{r}}\right|\right)$ with high dynamic capacity, and a second block that generates the rotor flux amplitude reference, in 

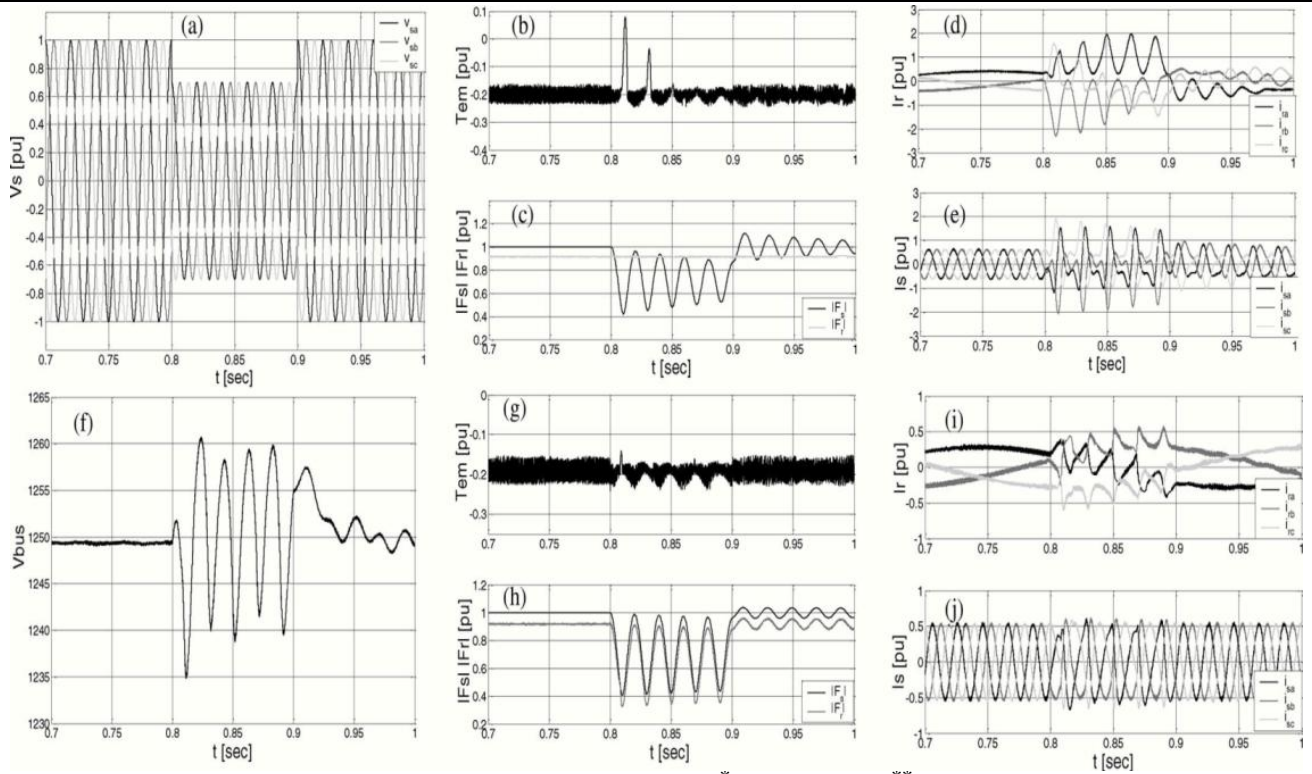

Fig. 2 Simulation comparison of DFIM behavior, without $\left(^{*}\right)$ and with $\left(^{* *}\right)$ proposed reference generation. (a) Stator voltage. (b) Torque*. (c) Stator and rotor fluxes*. (d) Rotor currents*. (e) Stator currents*. (f) DC bus

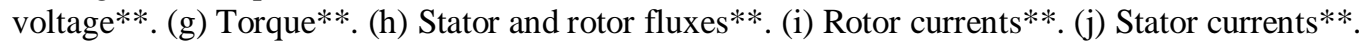

Order to handle with voltage dips. When the wind turbine is affected by a voltage dip, it will need to address three main problems:1) from the control strategy point of view, the dip produces control difficulties, since it is a perturbation in the winding of the machine that is not being directly controlled (the stator); 2) the dip generates a disturbance in the stator flux, making necessary higher rotor voltage to maintain control on the machine currents; and 3) if not the special improvements are adopted, the power delivered through the rotor by the back-to-back converter, will be increase of voltage and currents [2] in the rotor of the machine, provoking finally, an increase of the dc bus voltage [3].

Taking into account this, depending on the dip depth and asymmetry, together with the machine operation condition at the moment of the dip (speed, torque, mechanical power, etc.),implies that the necessity of the crowbar protection is inevitable in many faulty situations. However, in this letter, a control strategy that eliminates the necessity of the crowbar activation in some low depth voltage dips is proposed.

\section{Analytical Study}

When a voltage dip occurs, the stator flux evolution of the machine is imposed by the stator voltage equation

$$
\vec{v}_{s}^{s}=R_{s} \vec{l}_{s}^{s}+\frac{\overrightarrow{d \Psi_{s}^{s}}}{d t} .
$$

In general, since very high stator currents are not allowed the stator flux evolution can be approximated by the addition of a sinusoidal and an exponential term (neglecting $\mathrm{R}_{\mathrm{s}}$ )

$$
\begin{gathered}
\Psi_{\alpha s}=K_{1} e^{-k_{2 t}}+K_{3} \cos \left(\left(\omega_{s} t+K_{4}\right),\right. \\
\Psi_{\beta s}=K_{5} e^{-k_{2} t}+K_{3} \sin \left(\omega_{s} t+K_{4}\right) .
\end{gathered}
$$

Sinusoidal currents exchange with the grid will be always preferred by the application during the fault. It means that the stator and rotor currents should be sinusoidal.

However, by checking the expressions that relate the stator and rotor currents as a function of the fluxes

$$
\begin{gathered}
\vec{i}_{s}^{s}=\frac{\mathrm{L}_{\mathrm{h}}}{\sigma \mathrm{L}_{\mathrm{r}} \mathrm{L}_{s}}\left(\frac{L_{r}}{L_{h}} \vec{\Psi}_{s}^{s}-\vec{\Psi}_{r}^{s}\right), \\
\vec{i}_{r}^{s}=\frac{\mathrm{L}_{\mathrm{h}}}{\sigma \mathrm{L}_{\mathrm{r}} \mathrm{L}_{s}}\left(\frac{L_{s}}{L_{h}} \vec{\Psi}_{r}^{s}-\vec{\Psi}_{s}^{s}\right) .
\end{gathered}
$$

It is appreciated that it is very hard to achieve sinusoidal currents exchange, since only the rotor flux amplitudes controlled by a DTC technique.

Consequently, as proposed in next section, a solution that reasonably cancels the exponential terms from (3) is to generate equal oscillation in the rotor flux amplitude and in the stator flux amplitude. Finally, as it will be later shown that the quality of the currents is substantially improved with this oscillatory rotor flux, rather than with constant flux. 


\section{Rotor Flux Amplitude Reference Generation Strategy:}

As depicted from Fig. 3, the proposed rotor flux amplitude reference generation strategy, adds a term $\left(\Delta\left|\overrightarrow{\Psi_{r}}\right|\right)$ to the required reference rotor flux amplitude accordating to the following expression:

With $\left|\overrightarrow{\Psi_{s}}\right|$, the estimated stator flux amplitude and $\overrightarrow{\mid v_{s}} \mid$ voltage of the grid (not affected by the dip). This voltage can be calculated by several methods, for instance, using a simple small bandwidth low-pass filter, as illustrated in Fig.3. It must be highlighted that constants $\mathrm{K}_{1}-\mathrm{K}_{5}$ from (2) are not needed in the rotor flux reference generation reducing its complexity.

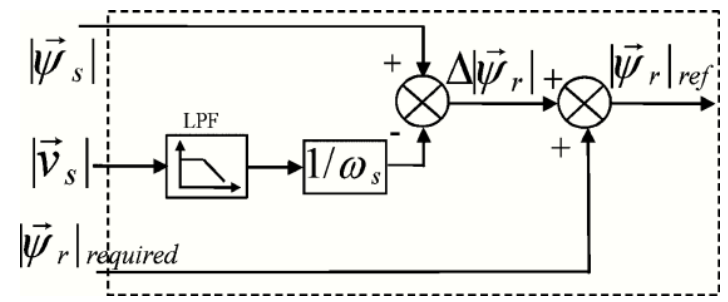

$$
\Delta\left|\overrightarrow{\Psi_{r}}\right|=\left|\overrightarrow{\Psi_{s}}\right|-\frac{\left|\vec{v}_{s}\right|}{\omega_{s}} .
$$

Fig .3 Rotor flux reference generation strategy

Note that at steady state without dips presence, the term $\Delta\left|\overrightarrow{\Psi_{r}}\right|$ will be zero. However, when a dip occurs, the added term to the rotor flux reference will be approximately equal to the oscillations provoked by the dip in the stator flux amplitude. For simpler understanding, the voltage drop in the stator resistance has been neglected.

\section{Simulation Results}

The simulated wind turbine is a $2 \mathrm{MW}, 690 \mathrm{~V}, N_{s} / N_{r}=1 / 3$ and two pair of poles DFIM. The main objective of this simulated validation is to show the DFIM behavior when a low depth [in this case $30 \%$, as illustrated in Fig.2 (a)] symmetric voltage dip occurs with and without the proposed flux reference generation strategy and at nearly constant speed. The simulations are performed in MATLAB/Simulink.

During the dip, it is desired to maintain the torque controlled to the required value (20\%), allowing to eliminate mechanical stresses to the wind turbine. This issue is achieved, as shown in Fig.2 (b) and (g), only if the oscillatory rotor flux is generated. For this purpose, the rotor flux is generated according to the block diagram of Fig.3, generating an equivalent oscillation to the stator flux amplitude [see Fig.2 (h)]. It must be pointed out that DTC during faults is a well-suited control strategy to reach quick flux control dynamics, as well as to dominate the situation, eliminating torque perturbations and avoiding mechanical stresses. Consequently, the proposed control schema maintains the stator and rotor currents under their safety limits, avoiding high over currents, as shown in Fig.2(i) and (j), either in the voltage fall or rise. However, as predicted in theory, it is hard to avoid deterioration of the quality of these currents. Nevertheless, if the rotor flux is maintained constant, the currents will go further till their limit values, as shown in Fig.2(d) and (e), provoking in a real case, a disconnection of the wind turbine or an activation of the crowbar protection. Moreover, by mitigating the over currents of the rotor, the back-to-back converter is less affected by this perturbation, producing short dc bus voltage oscillations, as illustrated in Fig.2(f).

Finally, it can be said that the proposed control is useful at any operating point of the wind turbine, as well as at any type of faults (one phase, two phases). The performance will be limited only, when the rotor voltage required is higher than the available at a given dc bus voltage.

\section{Conculsion}

Simulation results have shown that the proposed control strategy mitigates the necessity of the crowbar protection during low depth voltage dips. In fact, the dc bus voltage available in the back-to-back converter, determines the voltage dips depth that can be kept under control.

For future work, it would be interesting to explore the possibility to generate a modified reference of rotor flux and torque, in order to be able to address deeper voltage dips without crowbar protection.

\section{References}

[1] J. Lopez, E. Gubia , P. Sanchis, X. Roboam, and L. Marroyo, "Wind turbines based an doubly fed induction generator under asymmetrical voltage dips," IEEE Trans. Energy Convers., vol. 23, no.1, pp. 321-330, Mar, 2008.

[2] S.Seman, J.Niiranen, and A. Arkkio, " Ride-throug analysis of doubly fed induction wind-power generator under unsymmetrical network disturbance,” IEEE Trans. Power Syst., vol. 21, no. 4, pp. 1782-1789, Nov. 2006.

[3] X.Dawei, R. Li, P. J. Tavner, and S. Yang. "Control of a doubly fed induction generator in a wind turbine during grid fault ridethrough,” IEEE Trans. Eergy Convers., Vol. 21, no. 3, pp. 750-758, sep. 2006. 